\title{
Identification of novel biomarker and therapeutic target candidates for acute intracerebral hemorrhage by quantitative plasma proteomics
}

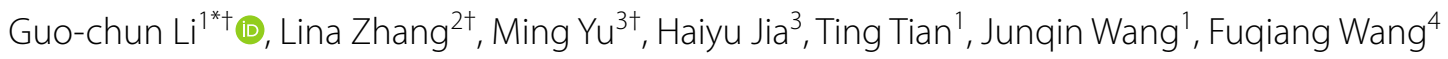
and Ling Zhou ${ }^{4^{*}}$

\begin{abstract}
Background: The systematic mechanisms of acute intracerebral hemorrhage are still unknown and unverified, although many recent researches have indicated the secondary insults. This study was aimed to disclose the pathological mechanism and identify novel biomarker and therapeutic target candidates by plasma proteome.

Methods: Patients with AICH $(n=8)$ who demographically matched healthy controls $(n=4)$ were prospectively enrolled, and their plasma samples were obtained. The TMT-LC-MS/MS-based proteomics approach was used to quantify the differential proteome across plasma samples, and the results were analyzed by Ingenuity Pathway Analysis to explore canonical pathways and the relationship involved in the uploaded data.

Results: Compared with healthy controls, there were 31 differentially expressed proteins in the ICH group $(P<0.05)$, of which 21 proteins increased while 10 proteins decreased in abundance. These proteins are involved in 21 canonical pathways. One network with high confidence level was selected by the function network analysis, in which 23 proteins, P38MAPK and NFKB signaling pathways participated. Upstream regulator analysis found two regulators, IL6 and TNF, with an activation z-score. Seven biomarker candidates: APCS, FGB, LBP, MGMT, IGFBP2, LYZ, and APOA4 were found. Six candidate proteins were selected to assess the validity of the results by subsequent Western blotting analysis.
\end{abstract}

Conclusion: Our analysis provided several intriguing pathways involved in $\mathrm{ICH}$, like LXR/RXR activation, acute phase response signaling, and production of NO and ROS in macrophages pathways. The three upstream regulators: IL-6, TNF, LPS, and seven biomarker candidates: APCS, APOA4, FGB, IGFBP2, LBP, LYZ, and MGMT were uncovered. LPS, APOA4, IGFBP2, LBP, LYZ, and MGMT are novel potential biomarkers in ICH development. The identified proteins and pathways provide new perspectives to the potential pathological mechanism and therapeutic targets underlying $\mathrm{ICH}$.

Keywords: Biomarkers, Acute intracerebral hemorrhage, Proteomics

\footnotetext{
*Correspondence: Dr.Guochun_li@njucm.edu.cn; Izhou@njmu.edu.cn ${ }^{\dagger}$ Guo-chun Li, Ming Yu and Lina Zhang contributed equally to this work. ${ }^{1}$ College of Medicine and Life Sciences, Nanjing University of Chinese Medicine, Nanjing 210023, People's Republic of China

${ }^{4}$ School of Public Health, Nanjing Medical University, Nanjing 211166, People's Republic of China

Full list of author information is available at the end of the article
} 


\section{Background}

Intracerebral hemorrhage ( $\mathrm{ICH})$ caused by ruptured cerebral blood vessels accounting for $10-20 \%$ of all strokes is the most devastating and the least treatable subtype of hemorrhagic stroke [1]. The 30-day mortality of $\mathrm{ICH}$ is reported to be $30-55 \%$, nearly half of deaths in the acute stage, especially in the first $48 \mathrm{~h}$, and approximately $80 \%$ of survivors are left with severe neurological deficits [2]. Compared with white populations of European origin, Chinese have a higher proportion of $\mathrm{ICH}$. However, the ongoing medical and surgical technological advances have not revealed an effective therapy for ICH. Therefore, it is urgent to explore the mechanisms underlying $\mathrm{ICH}$ and to identify the novel therapeutic targets so that we can improve the outcomes of ICH and develop new drugs for it.

Current researches have indicated that brain injury of $\mathrm{ICH}$ is primarily related to the secondary effects involving cerebral edema, inflammation autophagy, blood brain-barrier (BBB) disruption, cellular necrosis and apoptosis [3]. However, there has not been any confirmation study of ICH with human so far. Most experiments are undertaken with rodents, not entirely comparable to human ICH [4]. In addition, most people attempt to validate one or a few candidates rather than a panel of potential $\mathrm{ICH}$ biomarkers. In recent years, although gene expression profiling of perihematomal tissue after $\mathrm{ICH}$ has been performed in animal models as well as in human ICH samples [5], it should be noted that genomic expression and protein levels do not match in some cases [6]. Furthermore, proteins are the most informative biomolecules to advance the field, because not only are they the ultimate gene function performers, but also the direct manifestation of complexity and variability of life as well as the major drug targets of many diseases [6]. The proteins identified in recent researches provide essential information about the target proteins involved in multiple genetic interactions, with a wide range of clinical phenotypes in specific pathological pathways [7, 8]. Quantitative proteomics including isobaric tag for relative and absolute quantitation (iTRAQ) and tandem mass tag (TMT) have been a popular profiling approach in protein biomarker discovery and protein alterations quantification so far [9]. Quite a few researches have successfully obtained significant pathological insights once this technology was applied to brain tissue and cerebrospinal fluid (CSF) [10]. Collection of blood plasma, compared with tissue and CSF, is inexpensive and minimally invasive, and plasma proteins contain a wealth of information about the overall pathophysiological conditions of patients [11]. To date, no comprehensive proteomic analyses of plasma related to $\mathrm{ICH}$ has been reported. Therefore, a proteomics profiling of plasma in $\mathrm{ICH}$ will complete the pathological mechanisms of ICH and discover the therapeutic target candidates to monitor and improve the prognosis of this disease. In this follow-up study, a TMT-LC-MS/MS-based proteomics approach was used to quantify the differential proteome across plasma samples from $\mathrm{ICH}$ patients to explore possible mechanisms and potential therapeutic targets involved in $\mathrm{ICH}$.

\section{Methods}

\section{Ethics statement}

The Ethics Committee of Jiangsu Province Hospital of TCM (Affiliated Hospital of Nanjing University of Chinese Medicine) approved the study protocol. Written informed consent was given by all participates or legal guardians.

\section{Patient recruitment and sample collection}

A total of 12 people were invited to participate in this study. There were $8 \mathrm{ICH}$ patients and 4 demographically matched healthy controls (Table 1 ). The mean age of ICH patients and controls was 61.6 years (from 56 to 69 ) and 63.3 years (from 56 to 71 ) respectively, and $50 \%$ were male in both groups. The fasting blood samples were obtained from $8 \mathrm{ICH}$ patients on admission at Affiliated Jiangbin Hospital of Jiangsu University and Affiliated Hospital of Nanjing University of Chinese Medicine (Jiangsu Province Hospital of Traditional Chinese Medicine) in China between 2014 and 2015. The other 4 blood specimens were collected from healthy control subjects at the same time. The following inclusion criteria applied to the patients of the present study: the patients should be consistent with the diagnostic criteria of acute cerebral hemorrhage, admitted within $48 \mathrm{~h}$ with stroke onset, and willing and able to timely be referred in accordance with the requirements of the program. Patients were excluded if they were accompanied with transient cerebral ischemia, brain infarction or subarachnoid hemorrhage; their cerebral hemorrhage was caused by blood diseases, tumors or trauma; patients died within $24 \mathrm{~h}$ after admission or merged with serious primary diseases. Patients could not cooperate with the inspection because of disabilities included in the legislation framework.

Whole blood $(2 \mathrm{ml})$ was collected from study subjects. Altogether 12 plasma samples were collected, and $\mathrm{K}_{2}$-EDTA was used as the anti-coagulant agent. The blood samples were then centrifuged at $1500 \mathrm{~g}$ for $10 \mathrm{~min}$ at room temperature. The resulting plasma was aliquotted and stored at $-80^{\circ} \mathrm{C}$ before use. None of the samples were thawed more than twice before being analyzed.

\section{Plasma sample preparation and purification}

Since disease biomarkers in the blood, which are present at relatively low concentrations, are usually masked 
Table 1 Experimental protocol and basic characteristics in AICH patients $(n=8)$ and healthy controls $(n=4)$ group

\begin{tabular}{|c|c|c|c|c|c|c|c|}
\hline Block & Tissue & $\begin{array}{l}\text { Disease or } \\
\text { normal }\end{array}$ & $\begin{array}{l}\text { Patient } \\
\text { number }\end{array}$ & $\begin{array}{l}\text { Age/ } \\
\text { gender }\end{array}$ & $\begin{array}{l}\text { District } \\
\text { and hospital }\end{array}$ & $\begin{array}{l}\text { TMT labeling } \\
\text { reagent ID }\end{array}$ & $\begin{array}{l}\text { TMT labeling } \\
\text { code }\end{array}$ \\
\hline 1 & 1 & Normal & 1 & $66 / F$ & Nanjing & 1 & 126 \\
\hline 1 & 2 & $\mathrm{AlCH}$ & 2 & $61 / F$ & Nanjing & 1 & 127 \\
\hline \multirow[t]{2}{*}{1} & 4 & $\mathrm{AlCH}$ & 3 & $65 / F$ & Nanjing & 1 & 129 \\
\hline & & Reference & & & & 1 & 131 \\
\hline 2 & 6 & Normal & 4 & $60 / M$ & Nanjing & 2 & 126 \\
\hline 2 & 7 & $\mathrm{AlCH}$ & 5 & $56 / \mathrm{M}$ & Nanjing & 2 & 127 \\
\hline \multirow[t]{2}{*}{2} & 9 & $\mathrm{AlCH}$ & 6 & $58 / \mathrm{M}$ & Nanjing & 2 & 129 \\
\hline & & Reference & & & & 2 & 131 \\
\hline 3 & 11 & Normal & 7 & $71 / \mathrm{M}$ & Zhenjiang & 3 & 126 \\
\hline 3 & 12 & $\mathrm{AlCH}$ & 8 & $69 / M$ & Zhenjiang & 3 & 127 \\
\hline \multirow[t]{2}{*}{3} & 14 & $\mathrm{AlCH}$ & 9 & $66 / M$ & Zhenjiang & 3 & 129 \\
\hline & & Reference & & & & 3 & 131 \\
\hline 4 & 16 & Normal & 10 & $56 / F$ & Zhenjiang & 4 & 126 \\
\hline 4 & 17 & $\mathrm{AlCH}$ & 11 & $61 / F$ & Zhenjiang & 4 & 127 \\
\hline \multirow[t]{2}{*}{4} & 19 & $\mathrm{AlCH}$ & 12 & $57 / F$ & Zhenjiang & 4 & 129 \\
\hline & & Reference & & & & 4 & 131 \\
\hline
\end{tabular}

Reference represents a reference sample, i.e., a mixed sample of all plasma samples by internal standard method

AlCH acute intracerebral hemorrhage, $F$ female, $M$ male

by high-abundance proteins, and their signals are weak or even disappear in the mass spectrum [12,13], Agilent Human 14 Multiple Affinity Removal System (Agilent, USA) was utilized to deplete high-abundant proteins according to the manufacturer's instructions, including albumin, IgG, antitrypsin, IgA, transferrin, haptoglobin, fibrinogen, a2-macroglobulin, a1-acid glycoprotein, IgM, apolipoprotein AI, apolipoprotein AII, complement C3, and transthyretin. $100 \mu \mathrm{l}$ of depleted plasma from $\mathrm{ICH}$ or normal samples were added into $400 \mu \mathrm{l}$ of phosphatebuffered saline, $500 \mu \mathrm{l}$ of ice-cold acetone, $10 \%$ trichloroacetic acid (TCA) solution, and then precipitated at $-20{ }^{\circ} \mathrm{C}$. The resulting supernatant was removed and the pellets were washed thrice with ice-cold acetone and redissolved in $150 \mu \mathrm{l}$ of urea lysis buffer ( $8 \mathrm{M}$ urea, $75 \mathrm{mM}$ $\mathrm{NaCl}, 50 \mathrm{mM}$ Tris, pH 8.2, 1\% (V/V) EDTA-free protease inhibitor cocktail) and stored at $-80{ }^{\circ} \mathrm{C}$. The protein concentrations were measured by using the Bradford assay. Specific removal of 14 high-abundant proteins depletes approximately $94 \%$ of total protein mass from human plasma. The low-abundant proteins in the flow-through fractions can be studied. Removal of high-abundant proteins enables improved resolution and dynamic range for liquid chromatography/mass spectrometry (LC/MS).

\section{Protein digestion and TMT labeling}

One microgram of proteins from normal or ICH samples was reduced for $1 \mathrm{~h}$ at $60^{\circ} \mathrm{C}$ with $10 \mathrm{mM} \mathrm{DTT}$, and was alkylated with freshly prepared $55 \mathrm{mM}$ iodoacetamide (IAM) at room temperature in darkness for $40 \mathrm{~min}$.
Then, the proteins were digested with trypsin overnight at $37^{\circ} \mathrm{C}$ by the following buffer: $8 \mathrm{M}$ urea, $75 \mathrm{mM} \mathrm{NaCl}$, $50 \mathrm{mM}$ Tris, $\mathrm{pH} 8.2,1 \%$ (V/V) EDTA free protease inhibitor cocktail, and the tryptic peptides were desalted and dried in vacuo (Speed Vac, Eppendorf). Subsequently, $100 \mu \mathrm{g}$ of each sample was labeled with the reagent in accordance with the manufacturer,s protocol (Pierce, Rockford, IL, USA). After $2 \mathrm{~h}$ of TMT labeling, $5 \mu \mathrm{l}$ of $5 \%$ hydroxylamine was added to each tube and incubated at room temperature for $15 \mathrm{~min}$. Afterwards, the samples were mixed at the ratio of 1:1 based on total peptide amount, which was obtained by running an aliquot of the labeled sample in a conventional LC-MS/MS. Consequently, peptide expression of each reporter ion was normalized by the average intensity of the corresponding reporter ion (assuming the total intensities for each reporter ion were the same) in the data analysis process.

\section{Peptide fractionation with SCX chromatography}

Labeled peptide mixtures were reconstituted in solution $\left(10 \mathrm{mM} \mathrm{NH}_{4} \mathrm{COOH}, 5 \% \mathrm{ACN}, \mathrm{pH} 2.7\right)$ and fractionated by strong-cation exchange (SCX) columns $(1 \mathrm{~mm} \mathrm{ID} \times 10 \mathrm{~cm}$ packed with Poros 10 S, DIONEX, Sunnyvale, CA, USA), which were installed on the UltiMate ${ }^{\circledR} 3000$ HPLC system. The following two buffers carried out the separation. Mobile phase A was $5 \mathrm{mM}$ ammonium formate, $5 \% \mathrm{ACN}$ (pH 2.7); mobile phase $\mathrm{B}$ was $800 \mathrm{mM}$ ammonium formate, $5 \% \mathrm{ACN}$ (pH 2.7). The gradient for SCX was $0-30 \%$ B for $21 \mathrm{~min}, 30-56 \%$ B for $7 \mathrm{~min}, 56-100 \%$ B for $1 \mathrm{~min}$, $100 \%$ B for $3 \mathrm{~min}, 100-0 \%$ B for $1 \mathrm{~min}$ and $0 \%$ B for $20 \mathrm{~min}$ 
before the next run. Twenty fractions in total were collected from SCX separation and lyophilization.

\section{Mass spectrometry analysis}

The reversed phase chromatography was performed with a Nano ACQUITY UPLC system on the LTQ-Orbitrap instrument (Thermo Fisher, USA) via a nanospray source. The reversed-phase C18 column $(75 \mu \mathrm{m}$ i.d. $\times 15 \mathrm{~cm}$, $1.7 \mu \mathrm{m}, 130 \AA$ \& $\mathrm{BEH})$ was utilized with buffer A ( $2 \%$ ACN with $0.5 \%$ acetic acid) and buffer B (80\% ACN with $0.5 \%$ acetic acid), respectively. The 193 min linear gradient elution procedure was as follows: $4-9 \%$ buffer $B$ for $3 \mathrm{~min}, 9-33 \%$ buffer B for $170 \mathrm{~min}, 33-50 \%$ buffer B for 10 min, $50-100 \%$ buffer B for $1 \mathrm{~min}, 100 \%$ buffer B for 8 min, $100-4 \%$ buffer B for $1 \mathrm{~min}$. To analyze the proteins of plasma, eight most intense ions for collision-induced dissociation (CID) fragmentation were selected after mass spectrum scanning. CID was performed with 35\% normalized collision energy. The most intense ions from MS2 scan were selected and activated by using the higher energy collision-induced dissociation (HCD)-MS3.

\section{Protein identification and quantification}

The raw files obtained from precursor ions were analyzed with MaxQuant (version 1.2.2.5). Sequences search parameters were as follows: precursor mass tolerance was 20 parts per million (ppm); product ion mass tolerance was $0.5 \mathrm{Da}$; enzyme was trypsin with two maximum missed cleavages; cysteine carbamidomethylation $(+57.02146 \mathrm{Da}), \mathrm{N}$-terminus and lysine modification with TMT reagent adducts $(+229.162932 \mathrm{Da})$ were set as a fixed modifications; methionine oxidation $(+15.99492 \mathrm{Da})$ was set as a dynamic modification. The false discovery rates (FDR) of peptide and protein identification, which were estimated by searching against the database, were set $<1 \%$. Only peptides with at least six amino acids in length were considered to be successfully identified. The ratios of relative protein abundance between two groups were calculated by intensities of TMT reagent reporter ion from HCD spectra.

\section{Western blot analyses}

A standard western blotting analysis was used to investigate the protein expression levels after protein components were collected. Protein lysates from the plasmas of healthy controls $(\mathrm{n}=8)$ and $\mathrm{ICH}$ patients $(\mathrm{n}=8)$ were boiled in SDS-sample buffer for $5 \mathrm{~min}$ and then subjected to $8 \%$ SDS-polyacrylamidegel electrophoresis and transferred to PVDF (polyvinylidenefluoride) membranes (Bio-Rad). The membranes were then blocked for $2 \mathrm{~h}$ in 5\% milk-Tris-Buffered Saline Tween-20 (TBST) at room temperature, and incubated overnight at $4{ }^{\circ} \mathrm{C}$ either with the monoclonal antibodies (Cell Signaling Technology,
USA) of anti-APOA4, anti-IGFBP2, anti-LBP, anti-MGMT, anti-FGB or anti-APCS. The membranes were washed four times in TBST and incubated with the appropriate horseradish peroxidase-conjugated secondary antibodies at room temperature for $2 \mathrm{~h}$. Blots were visualized by enhanced chemiluminescence (Thermo, USA) and analyzed by a scanning densitometer with the molecular analysis software FluorChem M system (Protein Simple, USA) [14].

\section{Bioinformatics analysis Statistical analysis}

SPSS for Windows, Version 20.0 (Armonk, NY: IBM Corp.) was used for all statistical analyses. Univariate ANOVA (two-way analysis of variance with block design or one-way analysis of variance) was applied to compare the protein abundance among different groups. Protein abundance of control group was used for a reference to calculate the fold-change; changes of 1.3 or higher and the $P$ value $<0.05$ were considered significant.

\section{Ingenuity Pathway Analysis}

In order to further understand the biological significance of differentially expressed plasma proteins, Ingenuity Pathway Analysis (IPA; Ingenuity ${ }^{\circledR}$ Systems, www.Ingenuity.com/) was used to analyze canonical pathways and relationship of the uploaded data. Right-tailed Fisher's exact test was used to calculate a $P$ value to determine the significance of each canonical pathway, and $P$ value $<0.05$ was meaningful. Disease and functional protein networks and upstream regulator analysis with differently expressed proteins were presented, along with a Z-score. The Z-score $\geq 2$ or $\leq-2$ was considered significant activation or significant inhibition respectively.

\section{Results \\ Quantification of plasma proteins in healthy control and ICH samples}

Tandem mass spectrometry and TMT-labeled relative quantification proteomics were employed to screen differentially expressed proteins between ICH patients and healthy controls. The experimental workflow of this study is shown in Fig. 1. A total of 414 proteins and 3886 peptides were identified. Based on reporter ions, 411 proteins and 3165 peptides were quantified. The overall calibration factors and intensity distributions for all reporter ions were shown in Additional file 1: Figure S1. Compared with health control (NC) group, there were 31 differentially expressed proteins in $\mathrm{ICH}$ group (fold change $>1.2$ or fold change $<-1.2, P<0.05$ ), of which 21 proteins increased remarkably and the other 10 decreased in abundance (Table 2). The average fold-change for differentially expressed proteins between $\mathrm{AICH}$ and $\mathrm{NC}$ group was calculated to make a comparison. 


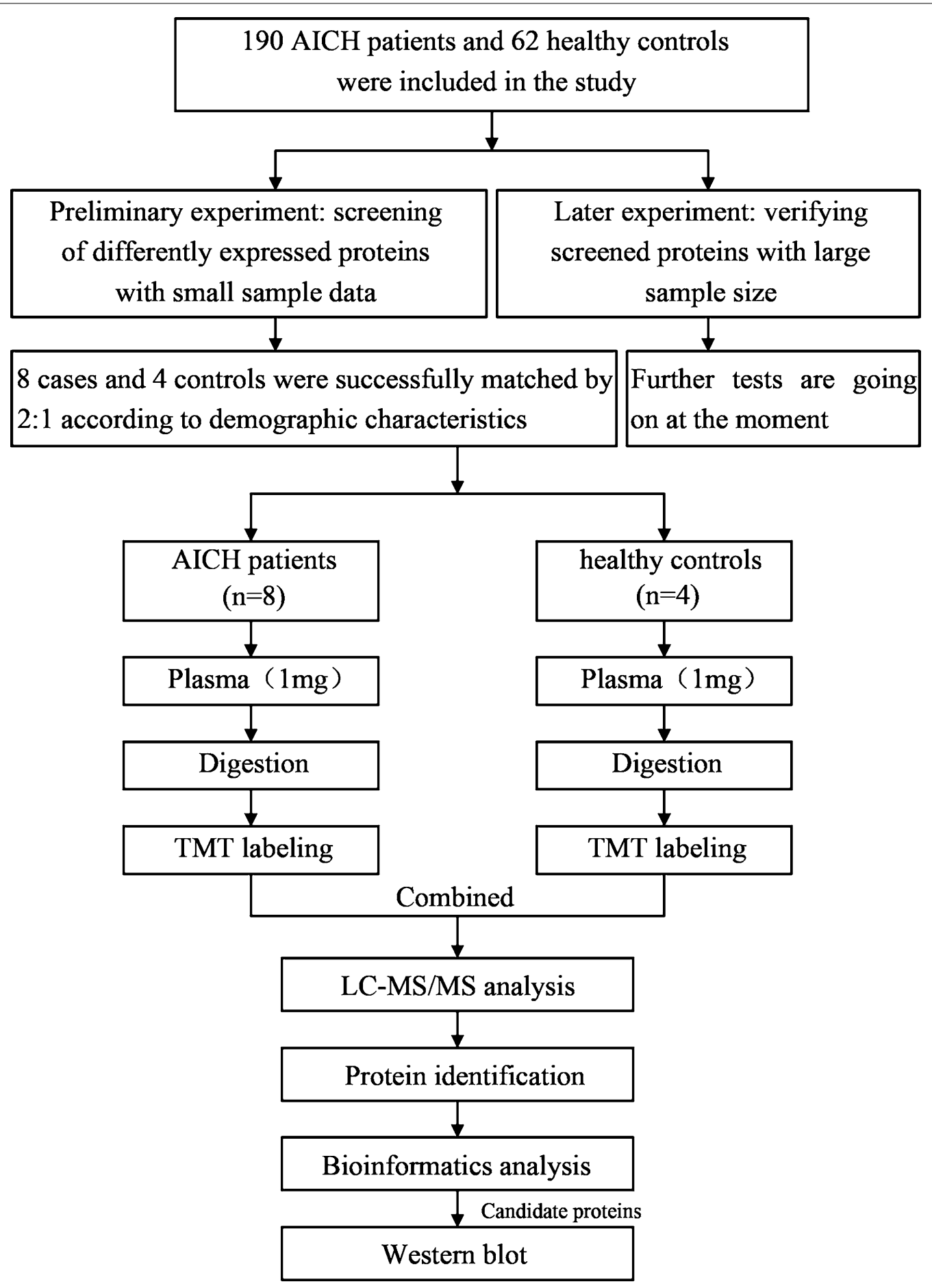

Fig. 1 The experimental profile

Gene ontology (GO) analysis

The Gene Ontology (GO) data were used to analyze the subcellular location and the function of the differentially expressed proteins. Figure 1 displays that 16 proteins (approximately 51.61\%) have been specified as extracellular space proteins. About $19.35 \%$ of the remaining proteins with subcellular location annotation were cytoplasm proteins, and $12.9 \%$ proteins were located in nucleus. The largest proportion of the differentially expressed proteins was represented by binding (41.9\%), followed by proteins with 


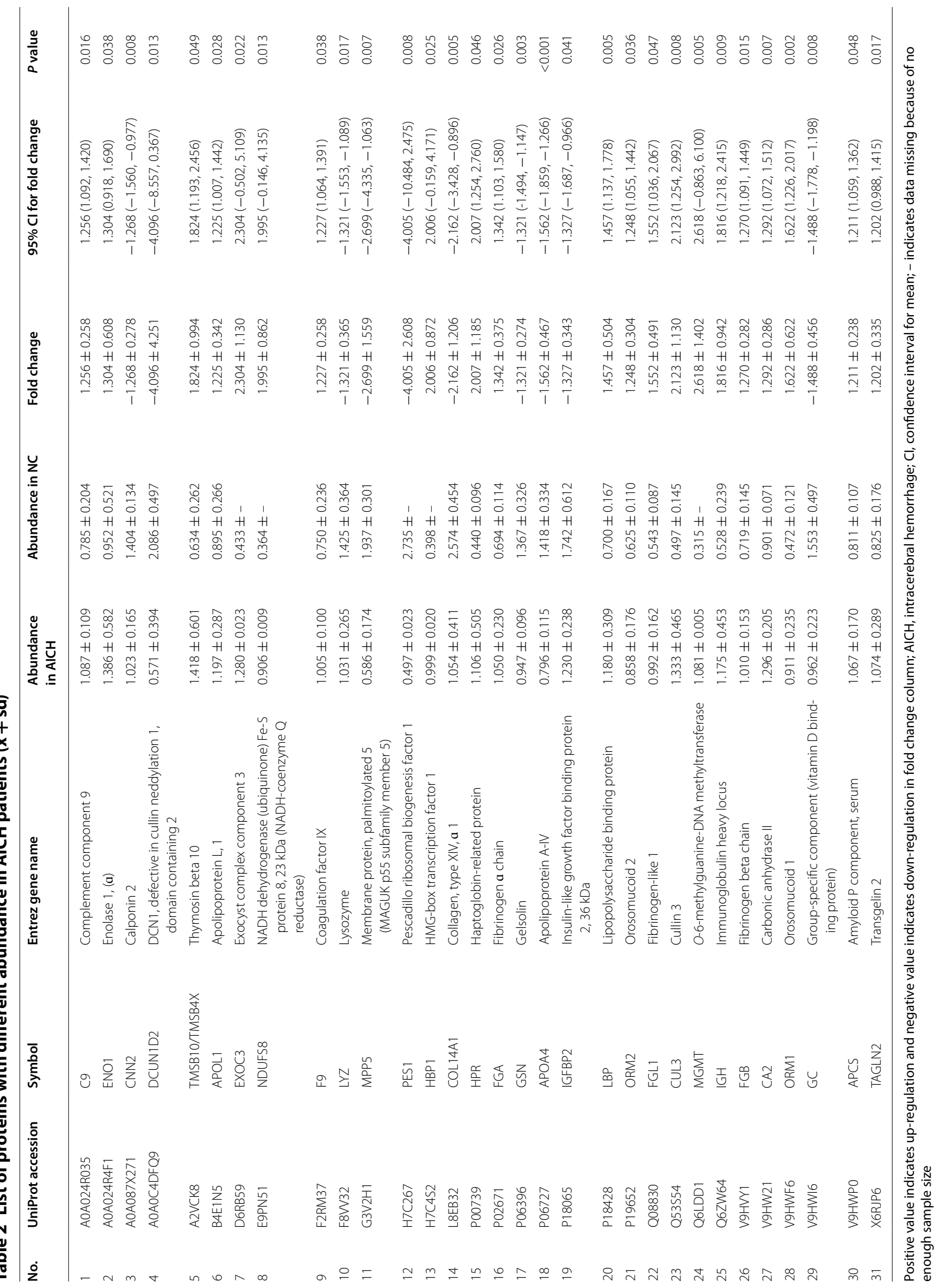


catalytic activity (32.3\%), transporter activity (12.9\%), and reporter activity (12.9\%). Regarding the biological process categories, the majority of proteins were involved in metabolic process $(45.2 \%)$, cellular process $(38.7 \%)$, response to stimulus (29.0\%), localization (29.0\%) and multicellular organismal process (19.4\%). Hence, extracellular space, binding, and cellular process were the major subcellular location, molecular function and cellular process of the proteins. And the overall functional distributions of the non-significantly expressed genes were similar to DE proteins (Additional file 2: Figure S2).

\section{IPA analysis of the proteins detected in ICH group}

In order to understand the biological significance of the 31 differentially expressed proteins in ICH better, the proteins were subjected to IPA software for further analysis of significantly involved molecular process. According to the Ingenuity Pathways Knowledge Base, IPA provided important information, such as canonical pathways, protein interaction networks, and upstream regulators. The canonical pathways along with up- and down-regulated proteins in each pathway were displayed in the bar chart of differentially expressed proteins. IPA analysis revealed that 10 proteins (APOA4, APOL1, C9, FGA, GC, HPR, LBP, LYZ, ORM1, ORM2) were in the positive Liver $\mathrm{X}$ receptor and retinoic acid $\mathrm{X}$ (LXR/RXR) activation pathway; 7 proteins (APCS, C9, FGA, FGB, LBP, ORM1, ORM2) in the positive acute phase response signaling pathway; 5 proteins (APOA4, APOL1, LYZ, ORM1, ORM2) in the positive production of nitric oxide
(NO) and reactive oxygen species in macrophages (ROS) pathway.

These proteins were also associated with functional network of metabolic diseases, cell-to-cell signaling and interaction. There were 23 proteins in the network (APCS, APLA4, APOL1, C9, CA2, COL14A1, CUL3, DCUN1D2, ENO1, FGA, FGB, GC, GSN, HBP1, HPR, IGFBP2, LBP, LYZ, MGMT, ORM1, ORM2, RES1, TMSB10/TMSB4X), and they were regulated by $\mathrm{p} 38$ Mitogen activated protein kinase (P38MAPK) as well as nuclear transcription factor $\kappa B(\mathrm{NF} \kappa \mathrm{B})$ (Fig. 2). The molecules in network and the score of network clustering were reported in Table 3.

Another meaningful research that we performed with IPA was upstream regulator analysis, which could predict the activation or inhibition of upstream regulators based on the differently expressed proteins identified in the present study so that the biological activities occurring in the ICH plasmas could be better understood. In our data, 99 upstream regulators of proteins have been discovered with a $P$ value $<0.05$, three of which were of activation $z$-score, including IL6 (z-score 2.026), TNF ( $z$-score 2.371), and lipopolysaccharide (LPS) ( $z$-score $2.219)$. They were considered to be target candidates for drug treatment. Theoretically, any chemical capable of inhibiting the expression of the three cytokines had the potential to treat $\mathrm{ICH}$ by preventing its progress and deterioration. The network analyses of the three upstream regulators and their target molecules for prediction in our dataset were shown in Fig. 3.
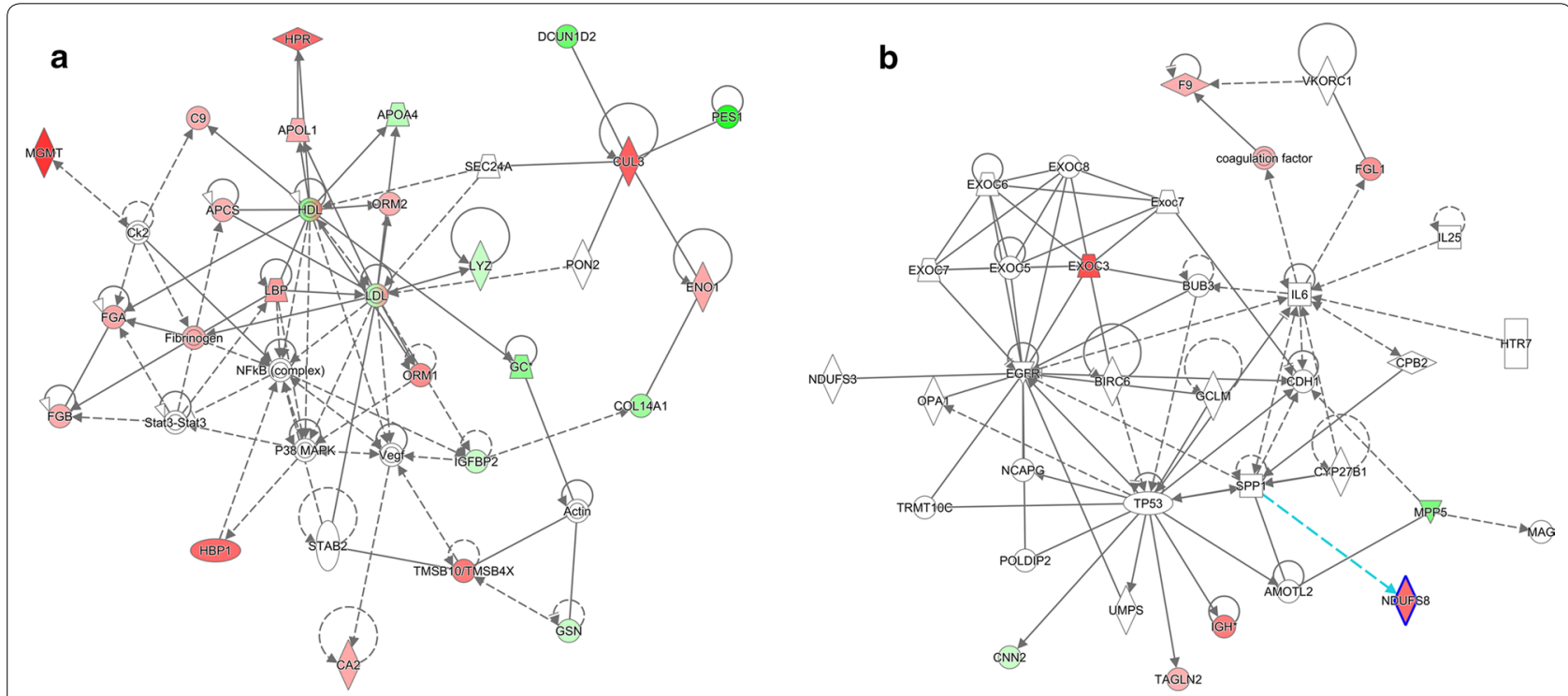

Fig. 2 The top 2 diseases and disorders networks identified by IPA analysis. a Metabolic disease, cell-to-cell signaling and interaction, renal damage network. b Cellular growth and proliferation, cancer, organismal injury and abnormalities network. Red and green indicate up-and down-regulation, respectively, of the proteins. Intensity of the color reflects the extent of differential expression. Dot lines represented indirect interaction; solid lines represented direct interaction; and arrows represented the direction of interaction 
Table 3 Major networks and associated proteins obtained by IPA analysis of differentially expressed ICH proteins

\begin{tabular}{|c|c|c|c|}
\hline Top diseases and functions & Molecules in network & Score & $\begin{array}{l}\text { Focus } \\
\text { molecules }\end{array}$ \\
\hline $\begin{array}{l}\text { Metabolic disease, cell-to-cell signaling } \\
\text { and interaction, renal damage }\end{array}$ & 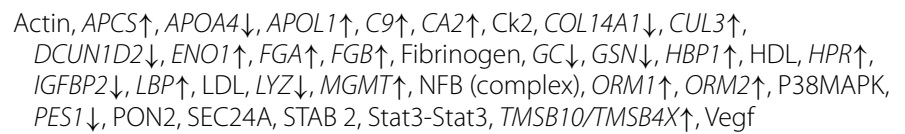 & 64 & 23 \\
\hline
\end{tabular}

The italicised proteins are identified in our data, and their expression is shown by arrows: up-regulation ( $\uparrow$ ) and down-regulation ( $\downarrow$ )

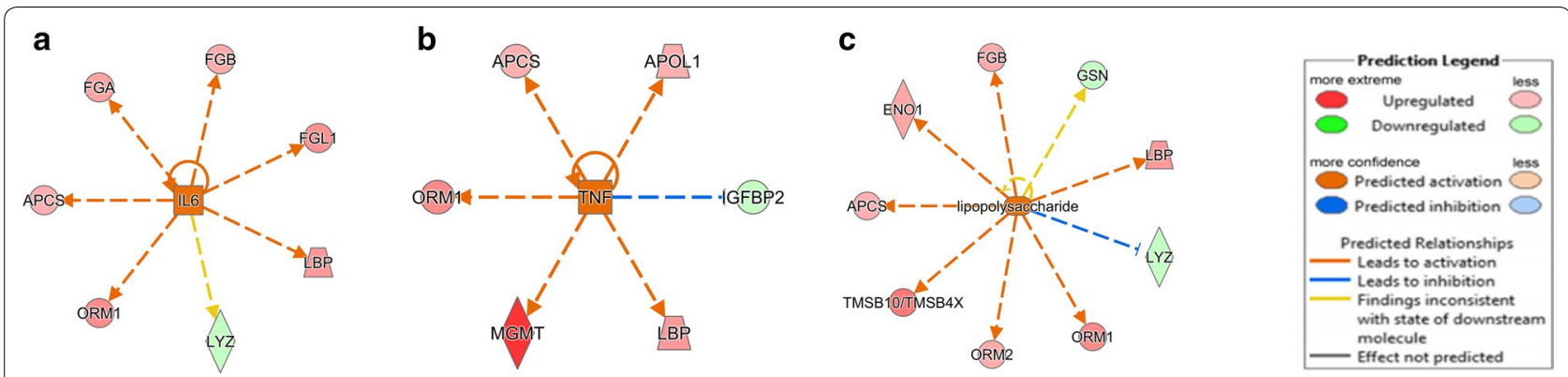

Fig. 3 Upstream regulator analysis of differentially expressed proteins in ICH. IL-6, TNF and LPS were predicted to be activated as determined by IPA. a, b, c represent 3 upstream regulators (IL-6, TNF and LPS) and their target molecules for prediction in our dataset respectively

To identify the potential biomarkers, we used the IPA biomarker filter to match the uploaded proteins with the list of biomarkers known for disease profiles. Some parameters settings in the IPA, with the related available options, were as follows: (1) Human species; (2) All molecule types; (3) Blood; (4) All biomarkers and disease applications (including cardiovascular disease, hematological disease, immunological disease, inflammatory disease, metabolic disease, and neurological disease). Seven proteins (APCS, APOA4, FGB, IGFBP2, LBP, LYZ, MGMT) were finally obtained as predicted markers for ICH shown in Table 4.

\section{Validation of proteomics results by Western blotting}

Western blotting analysis using GAPDH as an internal reference was performed for six important proteins (APOA4, IGFBP2, LBP, MGMT, FGB and APCS) to validate the iTRAQ results of the identified proteins.
Consistent with iTRAQ proteomics results, the intensity of bands by Western blotting of six proteins significantly changed in the plasma of AICH compared with healthy control subjects (Fig. 4, $P<0.05$ ).

\section{Discussion}

In this study, proteins extracted from plasmas of $8 \mathrm{ICH}$ patients and $4 \mathrm{NC}$ patients have been quantified by tandem-mass-TMT tagging labeling technology. Different expressions of 31 proteins in all were discovered, of which 21 were up-regulated, accounting for around $1 / 3$. As far as we know, this is the first comprehensive study to analyze ICH plasmas from each patient individually, in which high-throughput protein identification and quantification by MS were used to identify ICH biomarker.

Primary damage of $\mathrm{ICH}$ occurs within a few minutes to several hours since the onset of bleeding. It is mainly mechanical damage caused by hematoma with mass

Table 4 Selected candidate biomarkers by IPA biomarker filter analysis

\begin{tabular}{llllr}
\hline UniProt accession & Symbol & Entrez gene name & Location & Exp fold change \\
\hline V9HWP0 & APCS & Amyloid P component, serum & Extracellular space & 1.211 \\
P06727 & APOA4 & Apolipoprotein A-IV & Extracellular space & -1.562 \\
V9HVY1 & FGB & Fibrinogen beta chain & Extracellular space & 1.270 \\
P18065 & IGFBP2 & Insulin-like growth factor binding protein 2, 36 kDa & Extracellular space & -1.327 \\
P18428 & LBP & Lipopolysaccharide binding protein & Plasma membrane & 1.457 \\
F8VV32 & LYZ & Lysozyme & Extracellular space & -1.321 \\
Q6LDD1 & MGMT & O-6-methylguanine-DNA methyltransferase & Nucleus & 2.618 \\
\hline
\end{tabular}



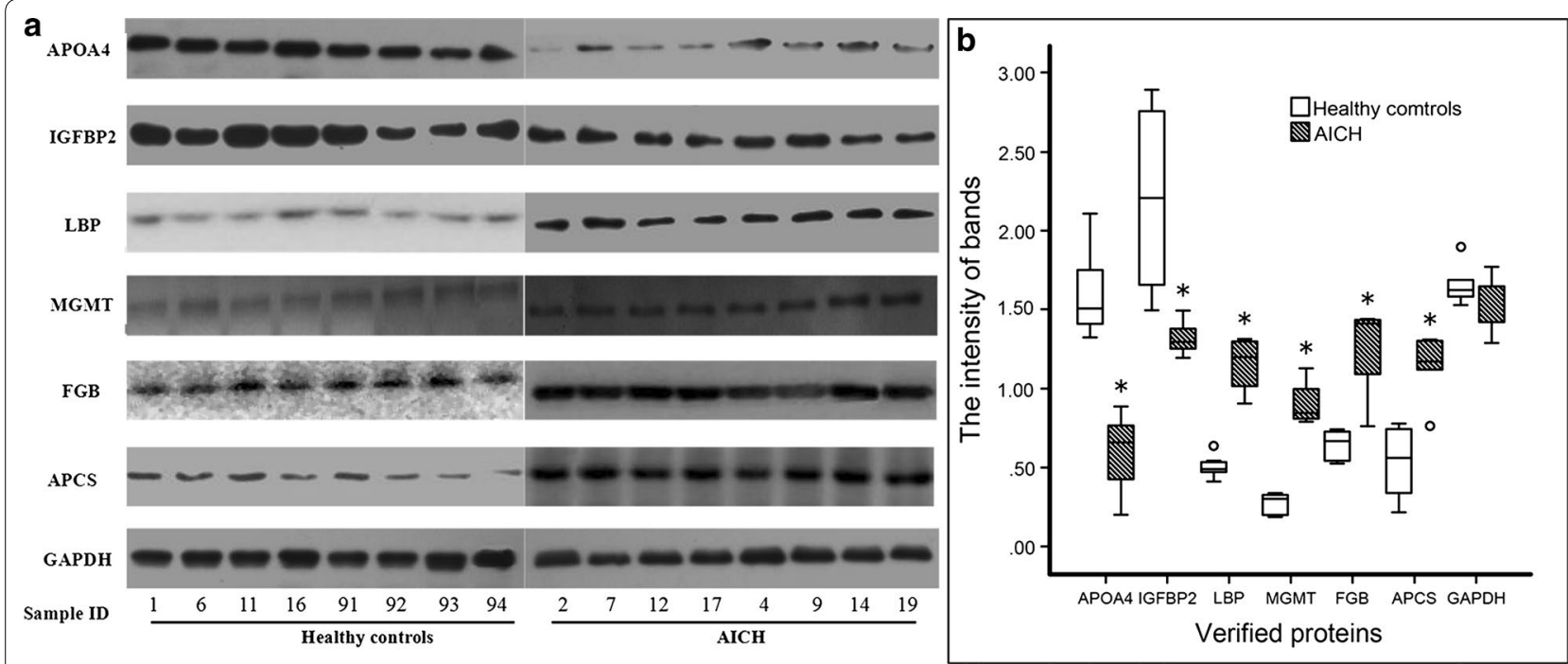

Fig. 4 Western blotting analysis of APOA4, IGFBP2, LBP, MGMT, FGB and APCS from healthy controls and AICH. GAPDH is the reference group by internal standard method. a Western blotting analysis of the six proteins. $\mathbf{b}$ The intensity of each band was measured using imaging analysis. $* P<0.05$ versus control

effect. However, secondary damage of $\mathrm{ICH}$, in most cases, is due to many parallel pathological pathways including: (1) cytotoxicity of blood, (2) hypermetabolism, (3) excitotoxicity, (4) spreading depression, (5) oxidative stress and inflammation. The canonical pathway analysis shows that 12 out of 31 proteins activate LXR/RXR pathway and participate in the acute phase response. They are the production of NO and ROS by macrophages, too. LXR/RXR is involved in the regulation of lipid metabolism, inflammation, cholesterol and bile acid catabolism [15]. Acute phase response is a rapid inflammatory response triggered by tissue injury, infection, or immunological disorders [16]. In the process of inflammation, the production of NO pro-inflammatory mediators in macrophages is attributed to the inducible form of nitric oxide synthase (iNOS). In addition, the microbicidal properties of macrophages are dependent on the production of reactive oxygen species (ROS). High level of NO and ROS causes oxidative stress, inflammatory reaction, eventually leading to tissue damage [17]. It has been reported that NO in ICH would result in secondary neuronal damage and aggravate tissue injury in the central nervous system, while ROS may be a major trigger of NLPR3 inflammasome activation following ICH $[18,19]$. The activation of three canonical pathways supports the momentous effect of inflammation in $\mathrm{ICH}$ as previous researches reported. Accordingly, we assume that the inflammatory response in $\mathrm{ICH}$ may be reduced effectively by inhibiting activation of the above signaling pathways.

IPA created an interaction network among the differentially expressed proteins, meanwhile, other signaling proteins revealed the interactions between disease and function. In the present study, these proteins are associated with metabolic diseases, cell-to-cell signaling and renal damage in which p38MAPK and NFKB are involved. Amazingly, no ICH related networks have been confirmed based on the existing IPA knowledge database, illustrating that the identified proteins have not been previously reported in a network with functions related to ICH. For all that, p38MAPK and NFKB are recommended as key regulators in the network, for it regulated the majority (23/31) of the proteins in this research. P38MAPKs, as one MAPK family member, is responsible for signal transmission between extracellular space to the nucleus, leading to biology reaction of cells [20]. A number of evidences demonstrate that p38MAPK signaling and NFkB are important inflammatory pathways in $\mathrm{ICH}$ through various mechanisms. Zhao et al. [21] found that p38MAPK and NFkB were activated in $24 \mathrm{~h}$ in animal model of $\mathrm{ICH}$, and the use of inhibitor could recede the inflammatory injury after $\mathrm{ICH}$. Meanwhile, the activation of NFKB in microglia/macrophages after ICH would result in the increase of pro-inflammatory cytokines such as TNF- $\alpha$ and IL-1 $\beta$ that might cause brain injury [22]. Together, p38MAPK and NFKB could represent potential therapeutic targets to prevent neurological deficits after $\mathrm{ICH}$.

IPA analysis found three upstream regulators activated based on the differently expressed proteins. IL- 6 is the key upstream regulator of 7 proteins, including FGA, FGB, FGL1, LBP, APCS, ORM1 and LYZ, because 6 out of the 7 proteins were activated by IL- 6 . TNF activated 5 proteins, 
including APCS, APOL1, ORM1, MGMT and LBP. LPS is an upstream regulator of 9 proteins, including FGB, ENO1, APCS, TMSB10/TMSB4X, ORM2, ORM1, LYZ and LBP, and IPA showed that 7 out of 9 proteins were activated by LPS. IL- 6 and TNF are cytokines that control differentiation, activation, apoptosis and other functions in many cell types [23]. As pro-inflammation cytokines, IL-6 and TNF play important roles in $\mathrm{ICH}$-induced inflammatory injury [24]. Immune system that is activated after $\mathrm{ICH}$ induces the release of numerous inflammatory cytokines, such as TNF- $\alpha$ and IL- 6 , and then the inflammatory responses damage brain tissue and kill plenty of brain cells [25]. Lei et al. [26] used the antibody of TNF to antagonize the inflammation response, which displayed that the antibody gave rise to palliating neuroinflammation and enhancing functional outcomes in a murine model of ICH. This study confirmed that IL-6 and TNF might be ideal drug targets for ICH. LPS is a form of endotoxin and has a significant effect on many diseases, such as septic shock, sepsis and Th2 inflammatory diseases [27], but its role in ICH has not been completely elucidated so far. LPS combined with CD14 would stimulate the release of inflammatory mediators from monocytes and endothelial cells, and finally cause systemic inflammatory response syndrome [28]. In this study, the upstream regulator of LPS activated 7 proteins shown with arrows in orange (Fig. 3c). The findings suggest that the discovery above is consistent with previous literatures, and LPS is indeed a good drug target candidate for ICH. But, it requires further experiments to test the effect of LPS on $\mathrm{ICH}$ and to validate the discovery of this study.

Identification of the putative biomarkers predicts 7 proteins as ICH markers by IPA. The level of 4 proteins (APCS, FGB, LBP, MGMT) increases, while three proteins (APOA4, IGFBP2, LYZ) decrease in ICH plasma samples. APCS is a glycoprotein, belonging to the pentraxin family. As a chaperone, it works on the binding of the encode protein to proteins in the pathological amyloid, and it increases amyloidosis of mice [29]. According to the report, the third etiological subcategory of $\mathrm{ICH}$ is the deposition of amyloid proteins in cerebral arteries, causing more blood vessels exudate [30]. Overexpression of APCS in ICH suggests that it may be an etiologic blood biomarker of ICH. FGB is the member of fibrinogen, cleaved by thrombin to form fibrin following vascular injury. Moreover, various cleavage products of fibrinogen and fibrin regulate cell adhesion, spreading and chemotactic activities, and they are mitogens of several cell types [31]. Leira et al. [32] have validated that the fibrinogen is an independent predictor of early neurologic deterioration in ICH patients. LBP is one of the acute phase immunologic response proteins for bacterial infection known to be involved in regulating LPS-dependent monocyte response [33]. As described above, the combination of LPS and CD14 can initiate inflammation response which is mediated by LBP, and many studies indicate that LBP is a marker of acute inflammation in patients with trauma [34]. In this study, the up-regulation of LBP suggests that it may be implicated in the acute inflammation response within $48 \mathrm{~h}$ after $\mathrm{ICH}$, thus it could be used as a candidate marker for ICH patients. MGMT is a DNA repair protein that plays a role in cellular defense against mutagenesis and toxicity from alkylating agents [35]. MGMT has been associated with several cancers like colorectal cancer and lung cancer [36], but its function in ICH has not been reported previously, so clinical experiments are needed to verify the results of this research.

Ideal biomarkers should be selectively and highly expressed in ICH patients [37]. However, APOA4, IGFBP2, and LYZ were down-regulated in ICH. APOA4 is a powerful activator of cholesterol transporter in vitro and is involved in antioxidant activity [36]. IGFBP2 is one of the six similar proteins that bind IGF-I and IGFII with high affinity. Its high expression level promotes the growth of several types of tumors [38]. LYZ is one of the antimicrobial agents found in human milk, kidney and tears. It is of antibacterial activity against a number of bacterial species. But the role of these proteins play in $\mathrm{ICH}$ has not been clear, thus further research is needed to elucidate the effect of the three proteins on $\mathrm{ICH}$.

Limitation in this research is the small sample size, although matching method that can reduce sample size is used to control confounding. We will expand the sample size in the following research. Small sample studies may be false negative, but the positive results of the protein are meaningful by verification.

\section{Conclusion}

Advances in neuroimaging and animal models have improved our understanding of the pathophysiology of ICH on molecular level. Most current theories imply a two-phase model of nerve damage: early mechanical damage phase and sub-acute phase characterized by inflammation and edema as response to hemorrhage. Proteomics analysis helped to find 31 plasma proteins which were differently expressed in acute ICH patients. Our analysis provided several novel pathways involved in $\mathrm{ICH}$, like LXR/RXR activation, acute phase response signaling, and production of $\mathrm{NO}$ and ROS in macrophages pathways. In the network analysis, p38MAPK and $N F \kappa B$ were known to implicate in progression and deterioration in the secondary damage phase of $\mathrm{ICH}$. In addition, we also discovered 3 upstream regulators including IL-6, TNF and LPS, as well as 7 biomarker candidates including APCS, APOA4, FGB, IGFBP2, LBP, LYZ and MGMT. Among them, IL-6, TNF, APCS, FGB 
have been identified to promote $\mathrm{ICH}$, but the remaining proteins are novel potential biomarkers which deserved further validation. Therefore, our data provided an unparalleled wealth of knowledge of ICH pathophysiology. The identified proteins and pathways provide new perspectives to the potential pathological mechanism and therapeutic targets underlying $\mathrm{ICH}$.

\section{Additional files}

Additional file 1: Figure S1. Calibration factors and overall intensity distributions for TMT labeling.

Additional file 2: Figure S2. The GO analysis of 31 differently expressed proteins and all other proteins quantified (those without differential abundance).

\section{Abbreviations}

AICH: acute intracerebral hemorrhage; ICH: intracerebral hemorrhage; TMT: tandem mass tag; CSF: cerebrospinal fluid; TMT-LC-MS/MS: two-dimensional-liquid chromatography tandem mass spectrometry (2D-LC-MS/MS) with tandem mass tagging (TMT); SCX: strong-cation exchange; CID: collision-induced dissociation; HCD: higher energy collision-induced dissociation; FDR: false discovery rates; IPA: Ingenuity Pathway Analysis; NC: normal controls; GO: gene ontology; NO: nitric oxide; ROS: reactive oxygen species in macrophages; p38MAPK: p38 mitogen activated protein kinase; NFkB: nuclear transcription factor KB.

\section{Authors' contributions}

GCL, LZ and LNZ conceived and designed the experiments, analyzed the data and drafted the manuscript. FQW performed the experiments and help to revise manuscript. MY and HYJ carried out the clinical diagnosis, recruited patients and helped to revise the manuscript. TT and JQW recruited patients, collected the blood samples and helped to revise the manuscript. All authors read and approved the final manuscript.

\section{Author details \\ ${ }^{1}$ College of Medicine and Life Sciences, Nanjing University of Chinese Medicine, Nanjing 210023, People's Republic of China. ${ }^{2}$ The Third Hospital of Zhangzhou, Zhangzhou 363005, People's Republic of China. ${ }^{3}$ Department of Neurology, Affiliated Hospital of Jiangsu University, Zhenjiang 212001, Peo- ple's Republic of China. ${ }^{4}$ School of Public Health, Nanjing Medical University, Nanjing 211166, People's Republic of China.}

\section{Acknowledgements}

We are grateful to Dr. Ye Yang, Yunan Zhao and Long Chen from Nanjing University of Chinese Medicine for critical review and helpful suggestions on the manuscript.

\section{Competing interests}

The authors declare that they have no competing interests.

\section{Consent for publication}

The authors showed their consent for publication of the research results.

\section{Ethics approval and consent to participate}

The research was approved by the Ethics Committee of Jiangsu Province Hospital of TCM (Affiliated Hospital of Nanjing University of Chinese Medicine) number of 2014NL-037-02 and complies with the guidelines of the Helsinki Declaration. Written informed consent for study participation was obtained from all participates or legal guardians.

\section{Funding}

This study was sponsored by The National Natural Science Foundation of China 2014 (81373512 to Li), Qing Lan Project and the Priority Academic Program Development of Jiangsu Higher Education Institutions (integration of Chinese and western medicine).

\section{Publisher's Note}

Springer Nature remains neutral with regard to jurisdictional claims in published maps and institutional affiliations.

Received: 18 January 2017 Accepted: 14 April 2017

Published online: 26 April 2017

\section{References}

1. D'Amore C, Paciaroni M, Silvestrelli G, Agnelli G, Santucci P, Lanari A, et al. Severity of acute intracerebral haemorrhage, elderly age and atrial fibrillation: independent predictors of poor outcome at three months. Eur J Intern Med. 2013;24:310-3.

2. Koivunen RJ, Satopaa J, Meretoja A, Strbian D, Haapaniemi E, Niemela M, et al. Incidence, risk factors, etiology, severity and short-term outcome of non-traumatic intracerebral hemorrhage in young adults. Eur J Neurol. 2015;22:123-32.

3. Ning M, Lopez M, Cao J, Buonanno FS, Lo EH. Application of proteomics to cerebrovascular disease. Electrophoresis. 2012;33:3582-97.

4. Steiner T, Petersson J, Al-Shahi SR, Christensen H, Cordonnier C, Csiba $L$, et al. European research priorities for intracerebral haemorrhage. Cerebrovasc Dis. 2011;32:409-19.

5. Lu A, Tang Y, Ran R, Ardizzone TL, Wagner KR, Sharp FR. Brain genomics of intracerebral hemorrhage. J Cereb Blood Flow Metab. 2006:26:230-52.

6. Guo T, Kouvonen P, Koh CC, Gillet LC, Wolski WE, Rost HL, et al. Rapid mass spectrometric conversion of tissue biopsy samples into permanent quantitative digital proteome maps. Nat Med. 2015;21:407-13.

7. Chiu CD, Chen TY, Chin LT, Shen CC, Huo J, Ma SY, et al. Investigation of the effect of hyperglycemia on intracerebral hemorrhage by proteomic approaches. Proteomics. 2012;12:113-23.

8. Ren C, Guingab-Cagmat J, Kobeissy F, Zoltewicz S, Mondello S, Gao M, et al. A neuroproteomic and systems biology analysis of rat brain post intracerebral hemorrhagic stroke. Brain Res Bull. 2014;102:46-56.

9. Tonack S, Aspinall-O'Dea M, Jenkins RE, Elliot V, Murray S, Lane CS, et al. A technically detailed and pragmatic protocol for quantitative serum proteomics using iTRAQ. J Proteomics. 2009;73:352-6.

10. Venugopal AK, Ghantasala SS, Selvan LD, Mahadevan A, Renuse S, Kumar P, et al. Quantitative proteomics for identifying biomarkers for Rabies. Clin Proteomics. 2013;10:3.

11. Liu Y, Buil A, Collins BC, Gillet LC, Blum LC, Cheng LY, et al. Quantitative variability of 342 plasma proteins in a human twin population. Mol Syst Biol. 2015:11:786.

12. Lochnit G, Geyer R. An optimized protocol for nano-LC-MALDI-TOF-MS coupling for the analysis of proteolytic digests of glycoproteins. Biomed Chromatogr. 2004;18:841-8.

13. Zelcer N, Hong C, Boyadjian R, Tontonoz P. LXR regulates cholesterol uptake through Idol-dependent ubiquitination of the LDL receptor. Science. 2009;325:100-4.

14. Zhang W, Wang F, Xu P, Miao C, Zeng X, Cui X, et al. Perfluorooctanoic acid stimulates breast cancer cells invasion and up-regulates Matrix Metalloproteinase-2/-9 expression mediated by activating NF-kB. Toxicol. Lett. 2014;229(1):118-25.

15. Heroux MS, Chesnik MA, Halligan BD, Al-Gizawiy M, Connelly JM, Mueller WM, et al. Comprehensive characterization of glioblastoma tumor tissues for biomarker identification using mass spectrometry-based label-free quantitative proteomics. Physiol Genomics. 2014;46:467-81.

16. DangLi RHWJL. ROS-induced ZNF580 expression: a key role for $\mathrm{H}_{2} \mathrm{O}_{2} /$ $\mathrm{NF}-\mathrm{KB}$ signaling pathway in vascular endothelial inflammation. Mol Cell Biochem. 2012;359:183-91.

17. Ma Q, Chen S, Hu Q, Feng H, Zhang JH, Tang J. NLRP3 inflammasome contributes to inflammation after intracerebral hemorrhage. Ann Neurol. 2014:75:209-19.

18. Wei $\mathrm{P}$, You $\mathrm{C}$, Jin $\mathrm{H}$, Chen $\mathrm{H}$, Lin B. Correlation between serum IL-1 $\beta$ levels and cerebral edema extent in a hypertensive intracerebral hemorrhage rat model. Neurol Res. 2014;36:170-5.

19. Ashraf Ml, Ebner M, Wallner C, Haller M, Khalid S, Schwelberger $\mathrm{H}$, et al. A p38MAPK/MK2 signaling pathway leading to redox stress, cell death and ischemia/reperfusion injury. Cell Commun Signal. 2014;12:6. 
20. Yang Z, Zhao T, Zou Y, Zhang JH, Feng H. Curcumin inhibits microglia inflammation and confers neuroprotection in intracerebral hemorrhage. Immunol Lett. 2014;160:89-95.

21. Zhou Y, Wang Y, Wang J, Anne SR, Yang QW. Inflammation in intracerebral hemorrhage: from mechanisms to clinical translation. Prog Neurobiol. 2014;115:25-44.

22. Sinn DI, Lee ST, Chu K, Jung KH, Kim EH, Kim JM, et al. Proteasomal inhibition in intracerebral hemorrhage: neuroprotective and anti-inflammatory effects of bortezomib. Neurosci Res. 2007;58:12-8.

23. Hsu TY, Hsieh TT, Yang KD, Tsai CC, Ou CY, Cheng BH, et al. Proteomic profiling reveals a 1 -antitrypsin, a 1-microglobulin, and clusterin as preeclampsia-related serum proteins in pregnant women. Taiwan J Obstet Gynecol. 2015;54:499-504.

24. Wang J, Dore S. Inflammation after intracerebral hemorrhage. J Cereb Blood Flow Metab. 2007;27:894-908.

25. Lei B, Dawson HN, Roulhac-Wilson B, Wang H, Laskowitz DT, James ML. Tumor necrosis factor a antagonism improves neurological recovery in murine intracerebral hemorrhage. J Neuroinflam. 2013;10:103.

26. Adib-Conquy M, Cavaillon JM. Stress molecules in sepsis and systemic inflammatory response syndrome. FEBS Lett. 2007;581:3723-33.

27. De Falco F, Di Giovanni C, Cerchia C, De Stefano D, Capuozzo A, Irace C, et al. Novel Non-Peptide small molecules preventing IKKbeta/NEMO association inhibit NF-kappaB activation in LPS-Stimulated $J 774$ macrophages. Biochem Pharmacol. 2016;104:83-94.

28. Togashi S, Lim SK, Kawano H, Ito S, Ishihara T, Okada Y, et al. Serum amyloid p component enhances induction of murine amyloidosis. Lab Investig J Techn Methods Pathol. 1997;77:525-31.

29. Manno EM. Update on intracerebral hemorrhage. Continuum (Minneap Minn). 2012;18:598-610.

30. Lominadze D, Tsakadze N, Sen U, Falcone JC, D'Souza SE. Fibrinogen and fragment D-induced vascular constriction. Am J Physiol Heart Circ Physiol. 2005;288:H1257-64.
31. Leira R, Davalos A, Silva Y, Gil-Peralta A, Tejada J, Garcia M, et al. Early neurologic deterioration in intracerebral hemorrhage: predictors and associated factors. Neurology. 2004;63:461-7.

32. Weber JR, Freyer D, Alexander C, Schroder NW, Reiss A, Kuster C, et al. Recognition of pneumococcal peptidoglycan: an expanded, pivotal role for LPS binding protein. Immunity. 2003;19:269-79.

33. Zeng $L, G u W$, Zhang $A Q$, Zhang M, Zhang $L Y, D u$ DY, et al. A functional variant of lipopolysaccharide binding protein predisposes to sepsis and organ dysfunction in patients with major trauma. Ann Surg. 2012;255:147-57

34. Takagi Y, Takahashi M, Sanada M, Ito R, Yamaizumi M, Sekiguchi M. Roles of MGMT and MLH1 proteins in alkylation-induced apoptosis and mutagenesis. DNA Repair (Amst). 2003;2:1135-46.

35. Word B, Lyn-Cook LJ, Mwamba B, Wang H, Lyn-Cook B, Hammons G. Cigarette smoke condensate induces differential expression and promoter methylation profiles of critical genes involved in lung cancer in $\mathrm{NL}-20$ lung cells in vitro: Short-term and chronic exposure. Int J Toxicol. 2013;32:23-31

36. Carter CJ. Convergence of genes implicated in Alzheimer's disease on the cerebral cholesterol shuttle: APP, cholesterol, lipoproteins, and atherosclerosis. Neurochem Int. 2007;50:12-38.

37. Lai $\mathrm{X}$, Chen S. Identification of novel biomarker and therapeutic target candidates for diagnosis and treatment of follicular adenoma. Cancer Genomics Proteomics. 2015;12:271-81.

38. Markart P, Faust N, Graf T, Na CL, Weaver TE, Akinbi HT. Comparison of the microbicidal and muramidase activities of mouse lysozyme $M$ and $P$. Biochem J. 2004;380:385-92.

\section{Submit your next manuscript to BioMed Central and we will help you at every step:}

- We accept pre-submission inquiries

- Our selector tool helps you to find the most relevant journal

- We provide round the clock customer support

- Convenient online submission

- Thorough peer review

- Inclusion in PubMed and all major indexing services

- Maximum visibility for your research

Submit your manuscript at www.biomedcentral.com/submit
O Biomed Central 